\title{
Review article: locomotion systems for ground mobile robots in unstructured environments
}

\author{
L. Bruzzone ${ }^{1}$ and G. Quaglia ${ }^{2}$ \\ ${ }^{1}$ University of Genoa, DIME, Via Opera Pia 15A, 16145 Genoa, Italy \\ ${ }^{2}$ Politecnico di Torino, DIMEAS, Corso Duca degli Abruzzi, 24, 10129 Turin, Italy
}

Correspondence to: L. Bruzzone (bruzzone@dimec.unige.it)

Received: 1 February 2012 - Revised: 7 June 2012 - Accepted: 19 June 2012 - Published: 12 July 2012

\begin{abstract}
The world market of mobile robotics is expected to increase substantially in the next $20 \mathrm{yr}$, surpassing the market of industrial robotics in terms of units and sales. Important fields of application are homeland security, surveillance, demining, reconnaissance in dangerous situations, and agriculture. The design of the locomotion systems of mobile robots for unstructured environments is generally complex, particularly when they are required to move on uneven or soft terrains, or to climb obstacles. This paper sets out to analyse the state-of-the-art of locomotion mechanisms for ground mobile robots, focussing on solutions for unstructured environments, in order to help designers to select the optimal solution for specific operating requirements. The three main categories of locomotion systems (wheeled $-\mathrm{W}$, tracked $-\mathrm{T}$ and legged $-\mathrm{L}$ ) and the four hybrid categories that can be derived by combining these main locomotion systems are discussed with reference to maximum speed, obstacle-crossing capability, step/stair climbing capability, slope climbing capability, walking capability on soft terrains, walking capability on uneven terrains, energy efficiency, mechanical complexity, control complexity and technology readiness. The current and future trends of mobile robotics are also outlined.
\end{abstract}

\section{Introduction}

The forecasts of all the major robotics research institutions clearly indicate that the world market of service robotics is expected to increase dramatically over the next $20 \mathrm{yr}$, surpassing the market of industrial robotics in terms of units and sales (International Federation of Robotics, 2012; Prassler and Kosuge, 2008; EURON, 2009; CCC and CRA, 2009); in particular, ground mobile robots are the most widespread category of service robots; $75 \%$ of total unit sales of professional service robots in 2010 were defence or field robots (International Federation of Robotics, 2012). Most mobile robots are designed to operate not only in structured environments but also in unstructured situations: important fields of application are homeland security (Murphy, 2004), surveillance (Quaglia et al., 2011), intervention in case of terrorist attacks (Birk and Carpin, 2006; Snyder, 2001), demining (Havlik, 2005; Cepolina and Hemapala, 2007), reconnaissance in dangerous situations (such as radioactive or chemical contamination; Hamel and Cress, 2001), agricul- ture (González et al., 2009; van Straten et al., 2002) and planetary exploration (Mishkin, 2004; Iagnemma and Dubowsky, 2004). Moreover, mobile robotics is a typical dual technology as it has significant military applications (Playter et al., 2006).

Several mechanical architectures of mobile robots have been proposed by academic and industrial researchers. These, of course, feature various combinations of advantages and drawbacks. Consequently, when conceiving a new mobile robot for a specific application, a designer has to evaluate an extensive range of possible technological solutions for its locomotion system, performing complex and time-consuming assessments. In the early design stages of a ground mobile robot, the expected operating environments must be analysed as these can belong to many different categories: indoor structured environments with flat and compact ground, with or without stairs; outdoor environments with differing terrain firmness, with or without obstacles, and so on. It is therefore useful to outline the variety of 
existing locomotion systems and to synthetically compare their strengths and weaknesses in different operating conditions.

This is the main aim of the work, which considers both research prototypes and commercially available industrial products. The analysis of state-of-the-art of locomotion systems for ground mobile robots has mainly focused on solutions for unstructured environments. As a matter of fact, many mobile robots for structured environments are quite simple from the mechanical point of view (in most cases, a layout with two active wheels featuring differential steering and one passive caster wheel is sufficient). Otherwise, when it is required to move on uneven or soft terrains or to climb obstacles, the mechanical design of the locomotion system is much more complex and many different schemes have been proposed; therefore, a synthetic comparison is more useful for designers.

It is very difficult to make a synthetic but exhaustive comparison of locomotion systems for ground mobile robots since the potential operating conditions are widely diversified (Siegwart and Nourbakhsh, 2004). An overview of legged robots is proposed in Machado and Silva (2006). In Seeni et al. (2008), a survey of the mobility concepts for extraterrestrial surface exploration is proposed with reference to a selection of qualitative and quantitative parameters; the same topic is discussed in Fiorini (2000).

A similar comparison is made in this paper, but with two main differences: the analysis does not only focus on the field of application of spatial exploration, and the four hybrid categories (legs-wheels - LW, legs-tracks - LT, wheels-tracks - WT, and legs-wheels-tracks - LWT) are discussed separately. The evaluation considers the following features: maximum speed, obstacle crossing capability, step/stair climbing capability, slope climbing capability, walking capability on soft terrains, walking capability on uneven terrains, energy efficiency, mechanical complexity, control complexity, and technology readiness. These features are defined in Sect. 3.

It should be pointed out that there is a vast amount of scientific literature concerning mobile robotics which cannot be exhaustively quoted or included in the References for reasons of space. The works that have been quoted were selected to synthetically represent and compare all locomotion system categories. Broader bibliographies are available in Siegwart and Nourbakhsh (2004); Campion and Chung (2008); Kajita and Espiau (2008); Kemp et al. (2008); Meyer and Guillot (2008).

\section{Classification of mobile robot locomotion systems and comparison methodology}

This paper considers locomotion systems for robots moving predominantly on the ground, even if some of them are capable of travelling on water for short distances (Altendorfer et al., 2001). Furthermore, special-purpose locomotion

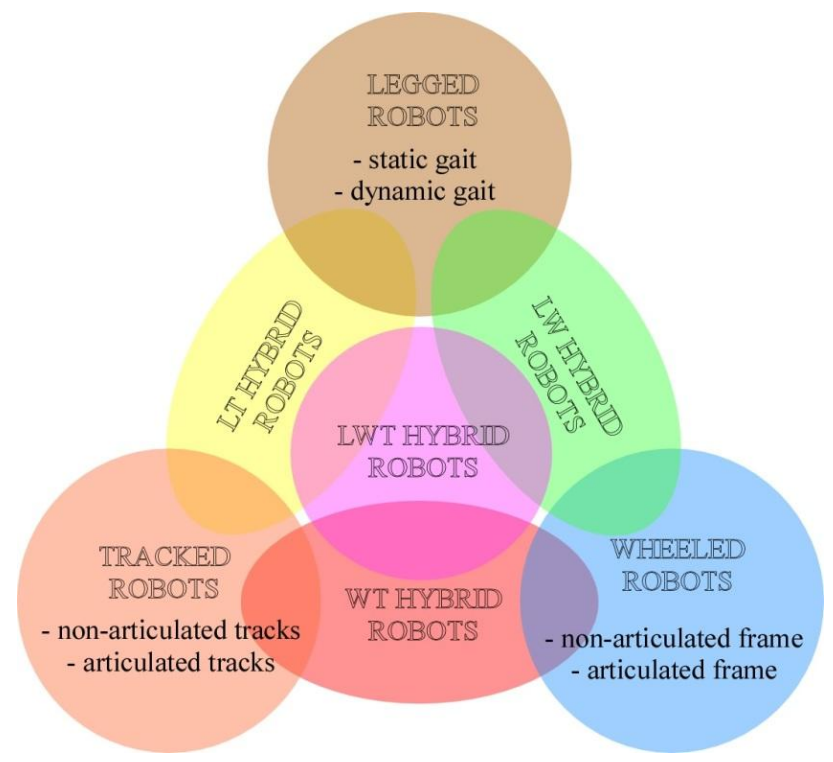

Figure 1. Ground mobile robot categories.

principles (e.g. jumping robots, snake-like slithering robots and wall-adhering robots) will not be discussed since they are devoted to very specific applications. Moreover, some researchers have proposed advanced locomotion strategies exploiting two or more co-operative mobile robots (Seeni et al., 2008); however, these co-operative approaches to robot mobility will not be considered since they require complex supervision systems and are rarely adopted; only locomotion principles for independent autonomous ground robots, not interacting with other vehicles or fixed devices, will be discussed.

On these premises, ground mobile robots can be classified into three main classes:

- wheeled robots $(\mathrm{W})$

- tracked robots $(\mathrm{T})$

- legged robots (L)

Moreover, there also exist robots featuring combinations of these locomotion principles, known as hybrid robots. In the scientific and industrial worlds, there are examples of the four possible combinations that lead to hybrid locomotion systems: legs-wheels (LW), legs-tracks (LT), wheelstracks (WT), and legs-wheels-tracks (LWT). The three main categories and the four hybrid categories of ground mobile robots are summarised in Fig. 1.

For a detailed comparison of locomotion systems, a set of features that can be objectively evaluated must be defined; to this end, a set of ten features is proposed in Table 1 together with their corresponding definitions.

The first seven features (maximum speed, obstacle crossing capability, step/stair climbing capability, slope climbing 
Table 1. Description of the features considered in the comparison of locomotion systems.

\begin{tabular}{ll}
\hline Feature & Definition \\
\hline maximum speed & $\begin{array}{l}\text { maximum speed on flat and } \\
\text { compact surfaces in the ab- } \\
\text { sence of obstacles }\end{array}$ \\
\hline obstacle crossing capability & $\begin{array}{l}\text { capability of crossing obsta- } \\
\text { cles with random shapes in } \\
\text { unstructured environments }\end{array}$ \\
(e.g. rocks) \\
\hline step/stair climbing capability & $\begin{array}{l}\text { capability of climbing up } \\
\text { single steps and stairs in } \\
\text { environments structured for } \\
\text { humans }\end{array}$ \\
\hline slope climbing capability & $\begin{array}{l}\text { capability of climbing com- } \\
\text { pact slopes with a sufficient } \\
\text { friction coefficient (>0.5) }\end{array}$ \\
\hline technology readiness & capability of walking on soft \\
and yielding terrains (e.g. \\
sand)
\end{tabular}

capability, walking capability on soft terrains, walking capability on uneven terrains, and energy efficiency) are related to pure mobility performance and are quantitatively measurable; the remaining three features (mechanical complexity, control complexity, and technology readiness) describe system complexity and influence other features, such as reliability, which are not expressly mentioned. Autonomy is not explicitly considered as it depends on energy efficiency.

\section{Mobile robot locomotion systems}

In this section, the main characteristics of the mobile robot locomotion system categories are summarised and compared with reference to the features defined in Table 1.

\subsection{Wheeled locomotion systems}

Wheeled robots can reach high speeds with low power consumption, and can be guided by controlling a few active degrees of freedom (Morin and Samson, 2008), but their ability to overcome obstacles is generally limited. Wheeled robots can be classified according to the number and position of the wheels. The minimum number of wheels to achieve static stability is three (the condition for static stability is that the vertical projection of the robot's centre of gravity on the ground must lie within the polygon formed by the wheelground contact points), through stability is improved with four or more wheels.

It should be noted that two-wheeled vehicles with an inverted pendulum layout have been developed for personal transportation (Liu and Parthasarathy, 2003), but in this case equilibrium is achieved in dynamic conditions by means of a complex control system and there is no substantial advantage with respect to three wheels if the overall height of vehicle and payload is not as relevant as in human transportation.

There are also examples of two-wheeled robots with inline configuration (robot motorcycles), but the mechanical model and the corresponding balance stabilisation and trajectory tracking control systems are even more complex than those governing the two-wheeled inverted pendulum scheme (Yi et al., 2006). Moreover, auxiliary legs or stabilisation rotors are required at zero-speed; for all of these reasons, unlike manned vehicles, this scheme is rarely adopted for autonomous robots.

Three-wheeled mobile robots with two differentially steered wheels and an idle caster wheel are widely used in structured environments with flat and even surfaces, for example, for transporting medicines in hospitals, because they require a simple control strategy and can spin around a vertical axis (Siegwart and Nourbakhsh, 2004). The same threewheeled architecture is used in commercial vacuum-cleaner robots such as Roomba by iRobot (www.irobot.com). On the other hand, this locomotion architecture is not suitable for unstructured environments because of its poor stability in the presence of uneven and sloping surfaces.

To achieve improved stability, most wheeled robots for unstructured environments usually have 4, 6 or 8 wheels. These layouts, however, are hyperstatic and require an articulated frame or suspensions to ensure all the wheels make contact with the ground.

Regardless of the number of wheels, it is possible to distinguish two categories of wheeled robots: with non-articulated frames and with articulated fames.

It should be pointed out that the second category only includes robots with passively articulated frames. If the wheels are carried by members in relative motion with respect to the main robot body, and this motion is actuated, these members are considered as legs and the robot becomes a leg-wheel hybrid (see Sect. 3.4.1). 


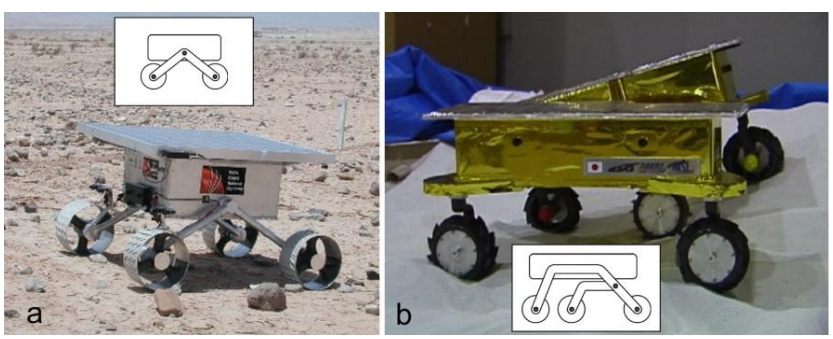

Figure 2. The four-wheeled SR2 rover (a, Miller et al., 2003) and the five-wheeled Micro5 rover (b, Kubotam et al., 2005).

In wheeled robots with non-articulated frames, the relative positions of the wheels are fixed, and with more than three wheels the configuration is hyperstatic and rarely adopted. Wheeled robots with articulated frames can adapt their configuration to the terrain, thanks to the mobility of the passive joints, and this reduces resistance while overcoming obstacles and irregularities.

The simplest possible mechanical design for wheeled robots for unstructured environments with four wheels is the $4 \times 4 \times 0$ wheel formula (wheel formula: total number of wheels $\times$ number of actuated wheels $\times$ number of actively steered wheels); the two wheels on each side are actuated by the same motor; steering is differential, achieved by setting different speeds on the two actuators. An example of this scheme is the SR2 rover developed by the University of Oklahoma for Malin Space Science Systems (Miller et al., 2003). The rover chassis is articulated in order to provide equal load distribution on the ground (Fig. 2a); this scheme allows pivotal turning, but the wheels slip on the ground while steering, thus reducing energy efficiency.

Where high speed is required, the robot locomotion system can be derived from automotive technology, adopting car suspensions and Ackerman steering geometry. Depending on the required mobile robot size, an unmanned vehicle can be created by fitting a car or another vehicle for human transport (usually four-wheeled) with an autonomous or remotelycontrolled navigation system. This is the approach used in the DARPA Grand Challenge, funded by the United States Department of Defense (Thrun et al., 2006). With this approach, of course, robot speed is very high but motion control accuracy and manoeuvrability in small areas is worse with respect to other electrically-actuated wheeled robots.

Mobile robots with five wheels are rare; the Micro5 rover, developed by the Japanese Developer Aerospace Exploration Agency (JAXA), features the $5 \times 5 \times 0$ wheel formula (Fig. 2b). The load is distributed by the suspension system known as the Pentad Grade Assist Suspension (PEGASUS) (Kubotam et al., 2005); its fifth wheel is connected centrally to the main body by a passive revolute joint. While climbing obstacles, the traction of the fifth wheel produces a nosedive momentum which increases the load on the front wheels and improves traction. This five-wheeled architecture was conceived to improve traction in applications with very low speeds (about $3 \mathrm{~cm} \mathrm{~s}^{-1}$ ) and low energy consumption, while it is not suitable for high speed operation due to the absence of suspensions.

Examples of six-wheeled robots with articulated frames are the rocker-bogie type rovers developed by NASA for Mars exploration, Spirit/Opportunity and Sojourner (Lindemann and Voorhees, 2005). These rovers feature the $6 \times 6 \times 4$ wheel formula (the front and rear wheels are independently steered). The two rocker-bogie mechanisms on each side of the vehicle (Fig. 3a) keep all the wheels in contact with the ground, even in presence of uneven terrain and obstacles. The average pressure on the wheels is passively equilibrated and this improves motion capability on soft terrains.

Other examples of wheeled robots with articulated frames are the SOLERO and CRAB rovers developed by the European Space Agency for the exploration of Mars (Michaud et al., 2002; Thueer et al., 2006a, b). SOLERO (Fig. 3b), based on the Shrimp mechanical design (Siegwart et al., 2002), features a $6 \times 6 \times 2$ wheel formula. The chassis has a bogie with two wheels on each side; the front wheel has a suspension system; the front and rear wheels are steered. CRAB II (Fig. 3c) is a rover with a $6 \times 6 \times 4$ wheel formula, comprising two symmetrical bogies on each side (the central wheel is connected to both bogies).

Eight-wheeled robots are quite rare. This scheme was used for the early lunar explorations (Lunokhod mission), but the latest space rovers are generally six-wheeled. Most robots with more than six wheels have an articulated frame with actively controlled degrees of freedom; therefore, they are considered as leg-wheel hybrid robots (see Sect. 3.4.1).

For the sake of completeness, it should be stated that most wheeled robots are fitted with deformable tyres (the larger sizes are usually inflatable while the smaller sizes are not) in order to reduce shocks while avoiding suspension systems. In some cases, omni-directional wheels (also known as Swedish wheels) are used to enhance robot mobility by reducing kinematic constraints while steering (Diegel et al., 2002). Nevertheless, these wheels are never adopted for unstructured environments as their mechanics can be damaged by shocks and dirt; moreover, conventional tyres give a better grip.

\subsection{Tracked locomotion systems}

Tracked robots are well suited to move on uneven and soft terrains and overcome obstacles, thanks to their large ground contact surface, but they move more slowly and consume more energy than wheeled robots: as a matter of fact, tracked robots are subject to vibrations as the lateral track profile is a polygon with moving vertices, and they are rarely fitted with damping systems. This limits maximum speed and reduces mechanical efficiency.

Tracked robots can be classified according to the number and layout of the tracks. First of all, robots can have non-articulated tracks or articulated tracks. If the relative 

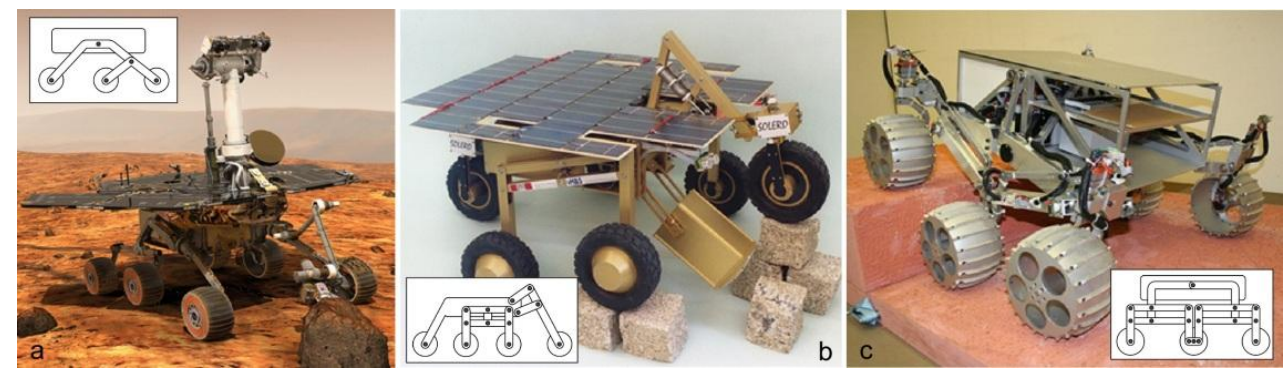

Figure 3. Six-wheeled rovers: rocker-bogie type (a, Lindemann and Voorhees, 2005), SOLERO (b, Michaud et al., 2002), CRAB II (c, Thueer et al., 2006a).

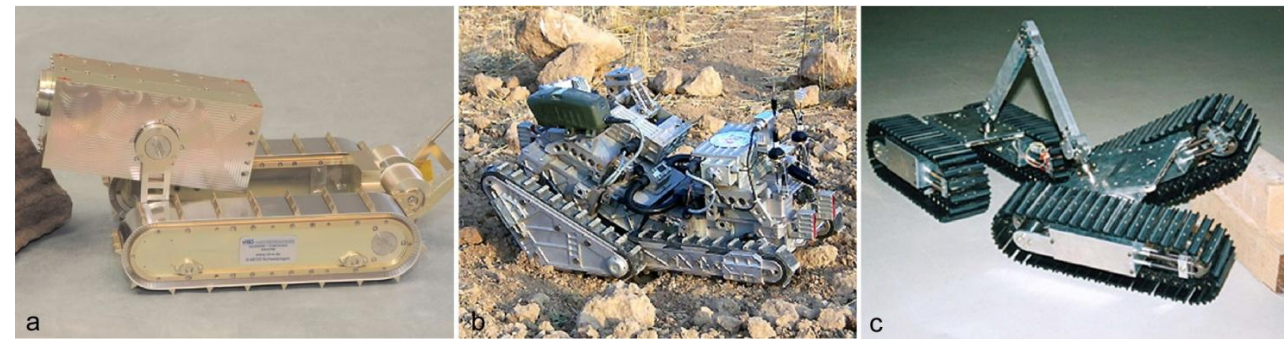

Figure 4. Tracked robots: Nanokhod (a, Klinker et al., 2007), Robhaz DT3 (b, Woosub et al., 2004) and Gunryu (c, Hirose et al., 1996).

mobility of the tracks is actuated, they are considered as leg-track hybrid robots (see Sect. 3.4.2). Robots with nonarticulated tracks feature very simple mechanics and controls. They generally have two parallel tracks with differential steering. In spite of their simplicity, they move well on uneven and soft terrains and over small obstacles. An example of this scheme is Nanokhod, the miniaturised space exploration tracker (Fig. 4a) based on Russian technology (Klinker et al., 2007). An example of an agricultural robot with two parallel tracks is Fitorobot (González et al., 2009), which is used for spraying in greenhouses.

To improve the capacity to handle uneven terrains and climb obstacles, more than two tracks with relative passive mobility can be adopted. For example, the Robhaz DT3 and DT5 are four-tracked robots developed by the KIST Intelligent Robotics Research Center (Woosub et al., 2004). They feature two parallel tracks with common actuation on each side, independently articulated with respect to the frame by revolute joints. The triangular shape of the forward tracks is designed to facilitate the approach to high obstacles (Fig. 4b).

To further enhance adaptability to uneven terrains and obstacles, other articulated tracked robots feature more complex mechanics. For example, Gunryu (Fig. 4c), developed by the Hirose-Fukushima Robotics Lab, has four independent tracks; the front and rear tracks are connected to two independent main bodies by revolute joints. Moreover, the two main bodies are connected by a passively articulated arm (Hirose et al., 1996). This configuration allows superior rel- ative track mobility and, consequently, a high capability of overcoming obstacles.

\subsection{Legged locomotion systems}

Legged robots have a broad mobility which makes them suitable for applications both in structured environments and on uneven terrains. However, they are relatively slow and consume a lot of energy. Generally speaking, legged robots have a large number of actuators and a complex control system.

The development of legged mobile robots is evidently biologically inspired: there exist robots with two legs (inspired by humans), with four legs (inspired by quadrupeds) and with more than four legs (inspired by insects). Though the most evident classification of legged robots is on the basis of the number of legs, their most important characteristic is the type of gait, which can either be static or dynamic.

Robots with static gait are always balanced, similarly to slow insects: their gait is planned so that the vertical projection of the robot's centre of gravity always remains within the polygon formed by the contact points of the legs with the ground. The gait is designed by assessing the quality of the robot's stability using proper stability indices (McGhee, 1985).

On the contrary, with a dynamic gait, the robot is not always balanced, similarly to fast walking, running, trotting or galloping animals (Raibert, 1986). Evidently, this type of locomotion requires a much more complex control system: 


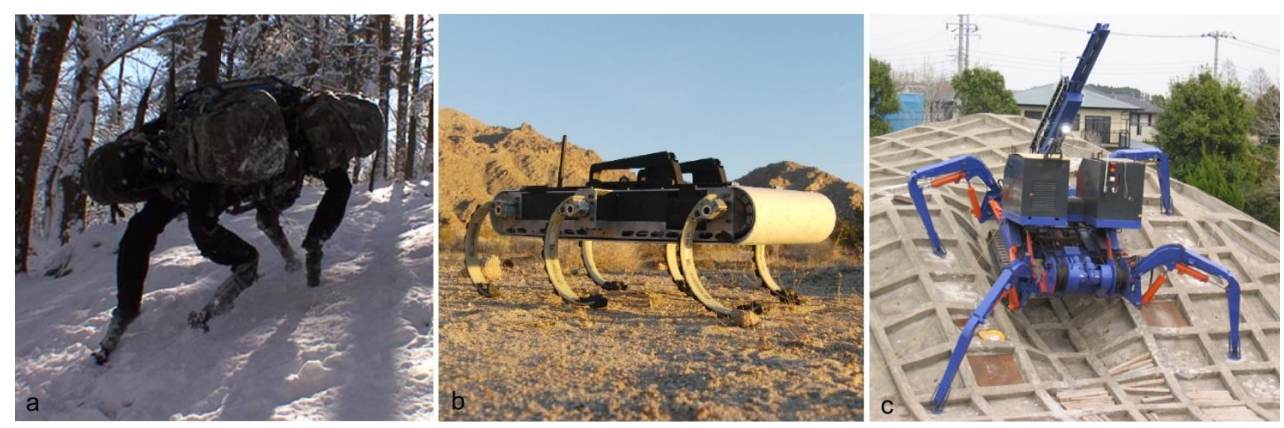

Figure 5. Legged robots: BigDog (a, Playter et al., 2006), RHex (b, Altendorfer et al., 2001), Titan XI (c, Hodoshima et al., 2007).

- the static gait design can only be based on the kinematic model, while the dynamic gait design must also be based on the dynamic model;

- with the static gait, different robot speeds can be achieved with the same leg motions performed at different speeds, while with the dynamic gait, leg trajectories must be modified as a function of the robot's speed;

- with the static gait, the robot can stop in any position and remain balanced, while with the dynamic gait, a proper movement must be performed in order to reach a rest position;

- with the dynamic gait, it is complex to compensate external disturbance forces and requires complete motion replanning.

Moreover, the increased complexity of the dynamic gait is not only related to control, but also to the mechanical architecture: for efficient dynamic walking, each robot leg should have several actuated degrees of freedom and, preferably, force sensors on its feet.

On the other hand, dynamic walking brings remarkable advantages. First of all, the energy efficiency is higher: with the static gait, the robot continuously uses power to change its configuration, but kinetic energy is lost at every step, while with the dynamic gait, most of the kinetic energy is conserved. Moreover, the dynamic gait can efficiently isolate the body from terrain irregularities and compensate external disturbance forces.

There are two main classes of model-based dynamic gait control strategies: the approaches based on the zero-moment point (ZMP) principle (Vukobratovic and Borovac, 2004) and those based on passive dynamic walking and limit cycle walking (McGeer, 1990; Manchester et al., 2011).

The basic idea of the ZMP approach is that the pressure centre remains within the polygon of the foot in contact with the terrain. The movements are conservative and stable but neither natural looking nor highly energy efficient. On the contrary, passive-dynamic and limit-cycle walkers are more life-like and energy efficient, as gravity and inertial effects have an important part to play in the generation of motion.
Moreover, strict ZMP walking does not admit a flight phase which characterises many dynamic movements, such as running or hopping. For all these reasons, many researchers consider the ZMP walking to be a quasi-static gait. On the contrary, passive-dynamic and limit-cycle walkers can perform motions with flying phases. In fact, they can be considered as biped or quadruped evolutions of the one-legged hopping robots by Raibert (1986) and Raibert et al. (1984).

Though discussion of these control techniques is out of the scope of this paper, it is important to note that the real-time implementation of these algorithms is the main obstacle to the realisation of legged robots with dynamic gait. Nevertheless, thanks to the progress made in microprocessor technology, legged mobile robots with advanced dynamic walking capability, agility and equilibrium comparable to human beings and animals have been developed during the last decade.

As regards biped locomotion, in the most advanced humanoid robots, the upper body and arms contribute to the movement, achieving a human-like dynamic gait with extended legs, while the first biped robots featured an unnatural gait with semi-bent legs. These robots can also soften impacts and stand up autonomously in case of tumbles due to strong unplanned external forces or slippery terrains (Hirukawa et al., 2005). They can also perform complex tasks such as dancing (Aucouturier et al., 2008).

Up until now, these state-of-the-art humanoid biped robots have mainly been developed and tested for research purposes, and their cost limits the range of possible applications. There is a growing number of commercially available smallscale humanoid robots, such as Nao by Aldebaran Robotics and Robonova by Hitec (http://www.aldebaran-robotics. com; http://www.robonova.de). Nevertheless, these robots are designed for edutainment and not for outdoor operations. On the contrary, quadruped robots with advanced dynamic walking capabilities, suitable for operations in highly unstructured environments, are already available. Bigdog by Boston Dynamics is a quadruped robot (height: $1 \mathrm{~m}$; length: $1 \mathrm{~m}$; weight: $90 \mathrm{~kg}$ ) powered by a diesel engine which drives a hydraulic actuation system (Fig. 5a). Each leg features three active degrees of freedom and one passive linear pneumatic 


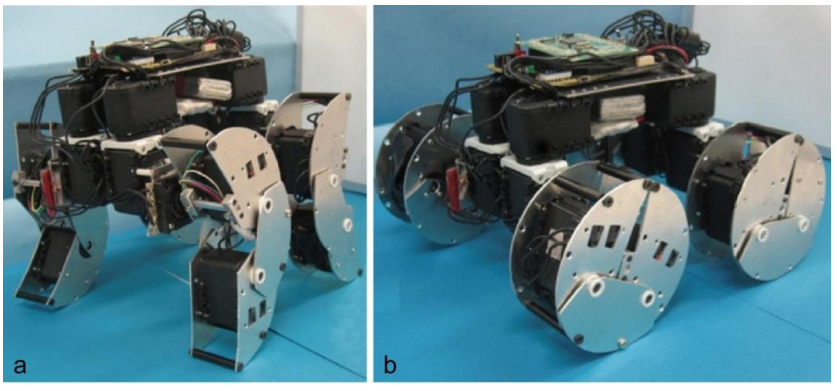

Figure 6. Leg-wheel hybrid robot with retractable locomotion modules: legged mode (a) and wheeled mode (b) (Tadakuma et al., 2010).

compliance in the lower leg. BigDog can walk and trot at speeds of up to $0.8 \mathrm{~m} \mathrm{~s}^{-1}$, and move on rough terrains and slopes up to $35^{\circ}$, while carrying a $50 \mathrm{~kg}$ payload (Playter et al., 2006).

In applications with small payloads, e.g. surveillance, robot size can be decreased. On this smaller scale, the complexity of the leg architecture and, consequently, control complexity can be reduced whilst preserving good motion performance. For example, RHex is a cockroach-inspired hexapod robot with compliant legs providing a self-stabilised gait, developed by a consortium of six American and Canadian universities (Fig. 5b). In spite of its simple mechanical design with one actuator per leg, RHex is capable of performing a wide variety of tasks, such as walking, running, leaping over obstacles and climbing stairs (Altendorfer et al., 2001). Another biologically inspired legged robot with simplified leg design is Whegs, developed by the Case Western Reserve University, which is fitted with three-spoke locomotion units (Quinn et al., 2003).

On the other hand, large legged robots for heavy payloads are usually controlled with static gait and move slowly in order to reduce the structural stresses caused by inertial effects. In this case, the most popular mechanical architecture is the quadruped. One example is Titan XI, developed by the Hirose-Fukushima Robotics Lab (Fig. 5c) for consolidating rocky slopes (mass: $7000 \mathrm{~kg}$; length of the hydraulically actuated legs: 3.7 m; Hodoshima et al., 2007).

\subsection{Hybrid locomotion systems}

Hybrid locomotion systems are probably the most interesting solutions for mobile robots as they combine the advantages of the various classes while attempting to avoid the drawbacks. In fact, legged locomotion is the most suitable solution in unstructured environments or in environments structured for humans (in presence of stairs, for example). On the other hand, humans often use wheeled or tracked extensions to increase their speed and energy efficiency. Unlike nature, in the design of a mobile robot, legs, wheels and tracks can be

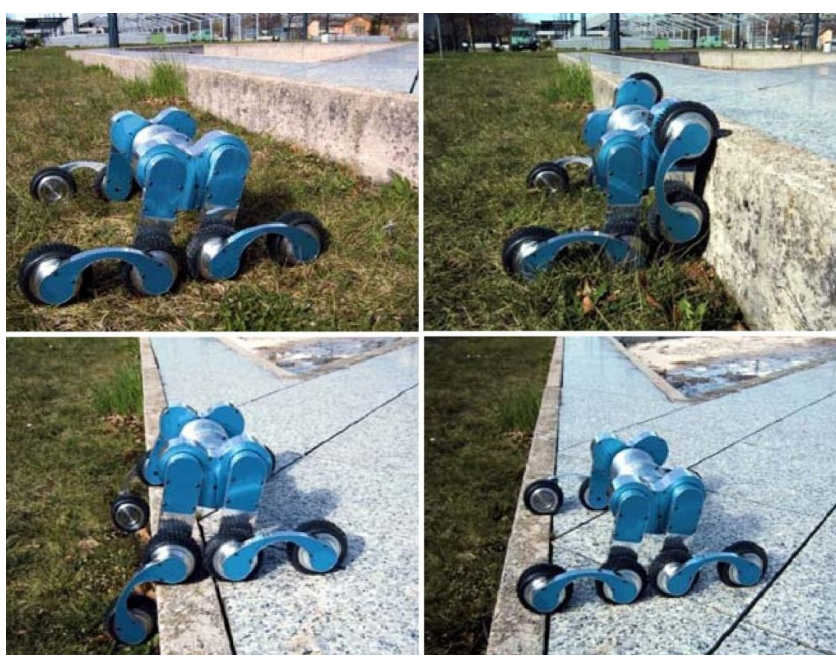

Figure 7. The leg-wheel hybrid Octopus robot (Lauria et al., 2002)

freely integrated. The four categories of hybrid mobile robots (LT, LW, WT, and LWT) are now discussed.

\subsubsection{Leg-wheel hybrid locomotion systems}

Leg-wheel robots combine the energy efficiency of wheels with the operative flexibility of legs. Legs and wheels can be basically combined in three ways (Tadakuma et al., 2010):

- fitting a wheeled robot with additional legs connected to the robot body;

- using retractable modules that can be used as wheels or legs;

- placing the wheels on the leg links (usually, but not always, at the ends of the legs).

The first approach is rarely adopted. Robot design is conceptually simple if legs and wheels are used alternatively, depending on terrain conditions. The main drawback is that the mass of the robot is usually high as the vehicle is fitted with two separate locomotion systems. Furthermore, if wheels and legs are used simultaneously, the advantage in terms of energy efficiency due to the wheels is significantly reduced.

The second approach is very interesting. For example in Tadakuma et al. (2010), a mobile robot with retractable legwheel modules is proposed (Fig. 6). The main drawback of this design is the mechanical complexity of the retractable modules and their low reliability in dirty environments or in case of shocks.

The third way of combining legs and wheels is probably the most effective. An example of a hybrid leg-wheel robot is Octopus (Fig. 7), developed by Ecole Polytechnique Fédérale de Lausanne (EPFL) (Lauria et al., 2002). It is fitted with tilt sensors and tactile wheels, while its sophisticated locomotion system has 8 motorised wheels and a total of 15 degrees 


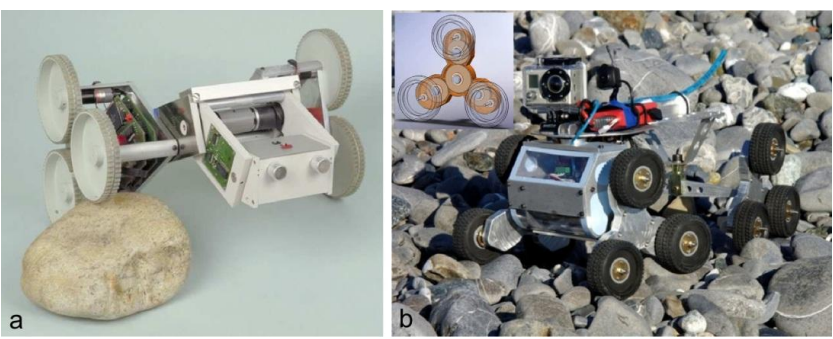

Figure 8. Stepping triple wheel hybrid robots: Spacecat (a, Siegwart et al., 1998); Epi.q (b, Quaglia et al., 2011).

of freedom which ensure great adaptability to uneven terrain and obstacle-climbing capability.

Another example of the third approach is the stepping triple wheel concept, introduced in the Spacecat microrover by EPFL (Siegwart et al., 1998): two three-wheeled locomotion modules can rotate independently with respect to the main body and allow the rover to actively lift one wheel to climb obstacles (Fig. 8a). Eight independent actuators are required for motion control: two for the locomotion modules and six for the wheels.

The same three-wheeled locomotion unit geometry, which assures good motion performance on uneven terrains and obstacle-climbing capability, is adopted in the Epi.q mobile robots family, developed in co-operation between the Polytechnic of Turin and the University of Genoa (Fig. 8b). Each Epi.q locomotion unit is underactuated by a single gearmotor through an epicyclical mechanism which automatically switches between legged locomotion and wheeled locomotion depending on dynamic and friction conditions, without control intervention. This significantly reduces the complexity of the control system (Quaglia et al., 2011).

\subsubsection{Leg-track hybrid locomotion systems}

Hybrid mobile robots with legs and tracks are fairly popular for difficult environments, provided that speed and energy efficiency are not crucial.

There are many ways of combining legs with tracks. The simplest approach is to use more than two tracks (usually four), which are in relative mobility with respect to the robot frame, in order to achieve legged locomotion. Examples of this approach are the commercially available mobile robots developed by iRobot for homeland security, surveillance, inspection, and explosive detection (Fig. 9a). These robots are extremely sturdy and reliable, with motion performance similar to tracked robots, but a greater capability of overcoming obstacles.

On the other hand, leg-track hybrid robots with more complex leg architectures have more extended mobility features and gait capabilities. Titan X, developed by the HiroseFukushima Robotics Lab (Fig. 9b), is a quadruped mobile robot with three degrees of freedom per leg; the four belts
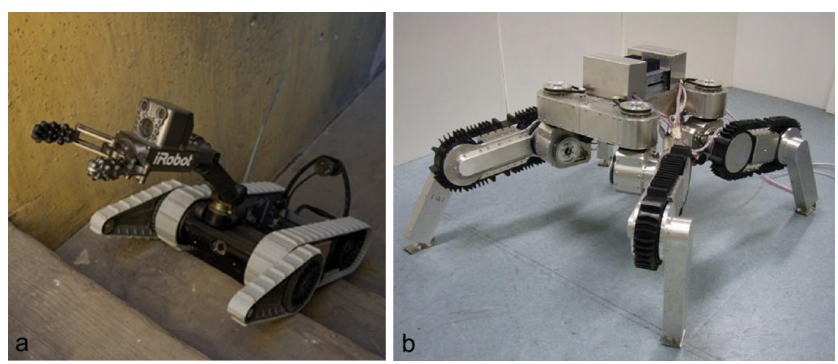

Figure 9. Leg-track hybrid robots: iRobot SUGV (a, http://www. irobot.com/gi/ground/) and Titan X (b, Hirose et al., 2009).

have a double function: mechanical transmission for actuation of the knee joints during legged locomotion, and tracks during tracked locomotion (Hirose et al., 2009). Another example of a leg-track hybrid robot is the one developed by a research group led by Yokota et al. (2006).

\subsubsection{Wheel-track hybrid locomotion systems}

The combination of wheels and tracks is very effective when good motion performance on soft and uneven terrains is required to be combined with energy efficiency on flat and compact artificial ground.

In wheel-track hybrid robots, the relative position of the tracks and wheels or the track shape can usually be changed to enable or disable wheel contact with the ground. For example, the Daegu Gyeongbuk Institute of Science \& Technology has developed a hybrid robot, featuring variable shape tracks (Fig. 10a) (Kim et al., 2010). In the configuration shown in Fig. 10, locomotion is achieved by the tracks; on flat compact ground, the tracks can be folded and locomotion obtained by the wheels.

On the contrary, in the Helios VI tracked carrier by the Hirose-Fukushima Robotics Lab (Fig. 10b), locomotion is never exclusively performed by the wheels; the two front wheels are added not to improve energy efficiency, but to increase stair climbing capabilities (Hirose et al., 2001).

Another interesting approach is the Galileo wheel patented system which, for example, is adopted in the VIPeR, a mobile platform for surveillance tasks developed by Elbit Systems (Fig. 10c). Thanks to extensible crawlers, the Galileo wheel combines wheels and tracks in a single group.

\subsubsection{Leg-wheel-track hybrid locomotion systems}

Azimuth is an example of a robotic platform that combines all three types of locomotion (Fig. 11); it is fitted with four independent leg-track-wheel articulations which can generate a wide variety of locomotion modes (holonomic and omni-directional motions, climbing of obstacles and stairs) (Michaud et al., 2005). Azimuth was mainly designed to perform in indoor environments, though the concept can be 

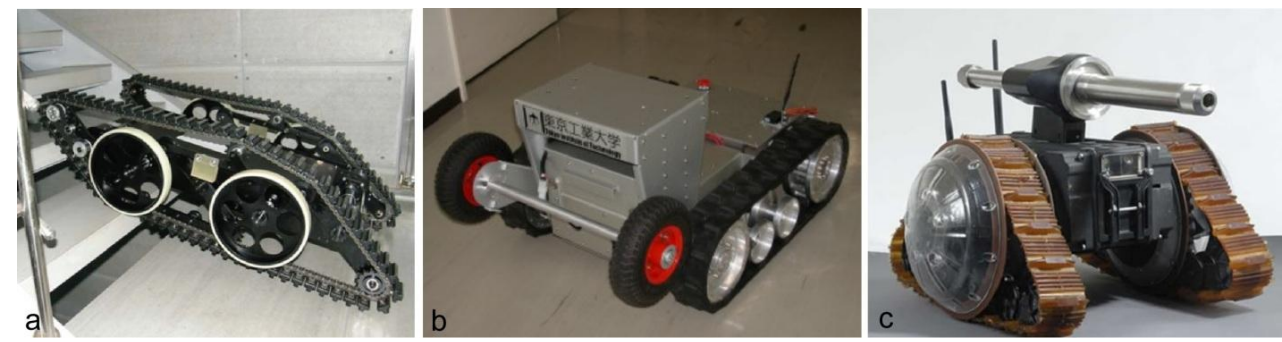

Figure 10. Wheel-track hybrid robots: with variable-shape tracks (a, Kim et al., 2010); Helios VI (b, Hirose et al., 2001); with Galileo wheel (c, http://www.galileomobility.com).

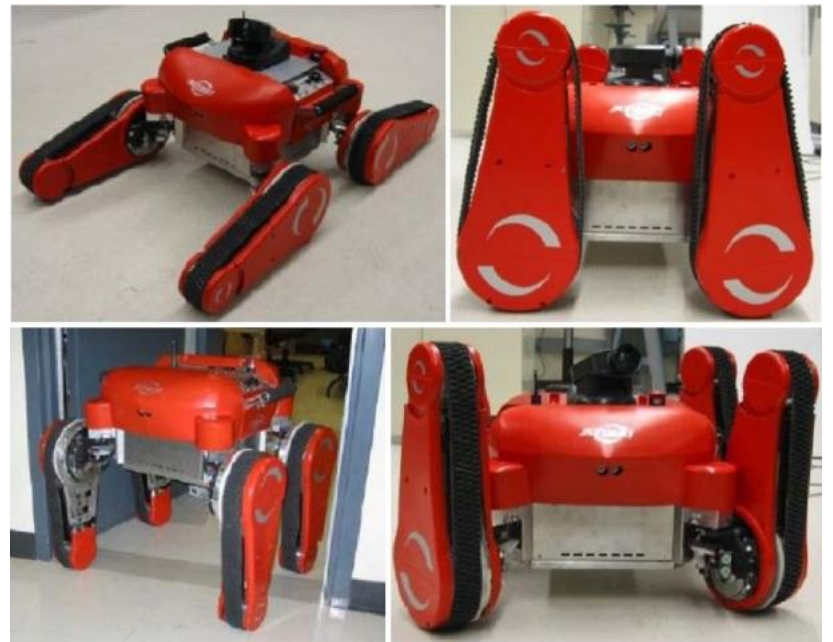

Figure 11. The hybrid leg-wheel-track Azimuth robot (Michaud et al., 2005).

adapted to outdoor settings. The main drawback is its high mechanical complexity.

\subsection{Comparison of locomotion system features}

With reference to the three main categories and the four hybrid categories of locomotion systems (Fig. 1), a very synthetic outline of locomotion system features is shown in Fig. 12, where mobility in unstructured environments $(y)$ vs. speed and energy efficiency $(x)$ is qualitatively graphed. Wheeled robots are in the right lower zone (better $x$, worst $y$ ); legged robots are in the left upper zone (worst $y$, better $x$ ); tracked robots are in the middle; hybrid combinations tend towards the right upper zone (combination of the benefits).

Along the $\mathrm{x}$-axis,

- legged robots are in the left zone as this type of locomotion implies impacts between feet and ground. Moreover, it requires torque delivery in the actuated joints, also in static conditions (unless non-reversible transmissions or elastic preload elements are used to balance the forces of gravity).

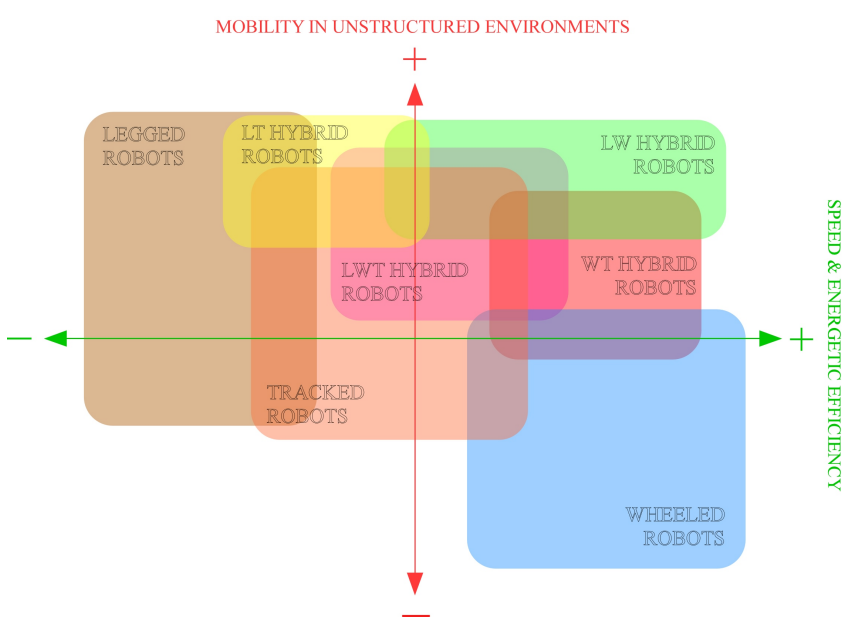

Figure 12. Comparison of locomotion systems.

- tracked robots are in the middle as the impacts with the ground are less significant but not absent. Moreover, the track elements are connected by several revolute joints with simple constructions and medium to poor energy efficiency.

- wheeled robots are in the right zone as they have few revolute joints (one per wheel), usually realised with ball bearings and therefore with a high energy efficiency.

Along the y-axis,

- wheeled robots are in the lower zone, because a wheel without additional devices cannot overcome obstacles if initial contact with the obstacle profile is not significantly lower than the wheel axis.

- tracked robots are in the middle as the large ground contact surface allows terrain unevenness to be filtered and contact pressure reduced.

- legged robots are in the upper zone, because this locomotion provides many degrees of freedom during motion in unstructured environments, since it allows the 
Table 2. Synthetic comparison of locomotion system features.

\begin{tabular}{|c|c|c|c|c|c|c|c|}
\hline $\begin{array}{l}\text { locomotion } \rightarrow \\
\text { feature } \downarrow\end{array}$ & Wheeled & Tracked & Legged & LW hybrid & LT hybrid & WT hybrid & LWT hybrid \\
\hline maximum speed & high & $\begin{array}{l}\text { medium/ } \\
\text { high }\end{array}$ & $\begin{array}{l}\text { low (static } \\
\text { walking) } \\
\text { to medium } \\
\text { (dynamic } \\
\text { walking) }\end{array}$ & $\begin{array}{l}\text { medium/ } \\
\text { high }\end{array}$ & medium & $\begin{array}{l}\text { medium/ } \\
\text { high }\end{array}$ & $\begin{array}{l}\text { medium/ } \\
\text { high }\end{array}$ \\
\hline $\begin{array}{l}\text { obstacle crossing } \\
\text { capability }\end{array}$ & low & $\begin{array}{l}\text { medium/ } \\
\text { high }\end{array}$ & high & $\begin{array}{l}\text { medium/ } \\
\text { high }\end{array}$ & high & medium & high \\
\hline $\begin{array}{l}\text { step/stair climbing } \\
\text { capability }\end{array}$ & low & medium & high & high & high & medium & high \\
\hline $\begin{array}{l}\text { slope climbing } \\
\text { capability }\end{array}$ & $\begin{array}{l}\text { low/ } \\
\text { medium }\end{array}$ & high & $\begin{array}{l}\text { medium/ } \\
\text { high }\end{array}$ & $\begin{array}{l}\text { medium/ } \\
\text { high }\end{array}$ & high & $\begin{array}{l}\text { medium/ } \\
\text { high }\end{array}$ & high \\
\hline $\begin{array}{l}\text { walking capability } \\
\text { on soft terrains }\end{array}$ & low & high & $\begin{array}{l}\text { low/ } \\
\text { medium }\end{array}$ & $\begin{array}{l}\text { low/ } \\
\text { medium }\end{array}$ & $\begin{array}{l}\text { medium/ } \\
\text { high }\end{array}$ & high & $\begin{array}{l}\text { medium/ } \\
\text { high }\end{array}$ \\
\hline $\begin{array}{l}\text { walking capability } \\
\text { on uneven terrains }\end{array}$ & low & $\begin{array}{l}\text { medium/ } \\
\text { high }\end{array}$ & high & high & high & $\begin{array}{l}\text { medium/ } \\
\text { high }\end{array}$ & high \\
\hline energy efficiency & high & medium & $\begin{array}{l}\text { low (static } \\
\text { walking) } \\
\text { to medium } \\
\text { (dynamic } \\
\text { walking) }\end{array}$ & $\begin{array}{l}\text { medium/ } \\
\text { high }\end{array}$ & medium & $\begin{array}{l}\text { medium/ } \\
\text { high }\end{array}$ & $\begin{array}{l}\text { medium/ } \\
\text { high }\end{array}$ \\
\hline $\begin{array}{l}\text { mechanical } \\
\text { complexity }\end{array}$ & low & low & high & $\begin{array}{l}\text { medium/ } \\
\text { high }\end{array}$ & medium/ high & $\begin{array}{l}\text { low/ } \\
\text { medium }\end{array}$ & high \\
\hline control complexity & low & low & high & $\begin{array}{l}\text { medium/ } \\
\text { high }\end{array}$ & medium/ high & medium & high \\
\hline $\begin{array}{l}\text { technology } \\
\text { readiness }\end{array}$ & full & full & $\begin{array}{l}\text { full for } \\
\text { static } \\
\text { walking; } \\
\text { in progress } \\
\text { for } \\
\text { dynamic } \\
\text { walking }\end{array}$ & $\begin{array}{l}\text { full (usu- } \\
\text { ally static } \\
\text { walking) }\end{array}$ & $\begin{array}{l}\text { full (usually } \\
\text { static walking) }\end{array}$ & full & $\begin{array}{l}\text { full (usually } \\
\text { static walk- } \\
\text { ing) }\end{array}$ \\
\hline
\end{tabular}

contact points between feet and terrain/obstacles to be selected.

In general, hybrid locomotion systems should combine the benefits of the different categories from which they derive. Therefore, in the graph in Fig. 12, each hybrid category should be located in an area characterised by the maximum values of the original categories along each axis. Nevertheless, this does not occur completely (and the graph is drawn accordingly), because the combination of alternative locomotion devices lowers overall performance: in fact, a nonactive locomotion device is a non-negligible payload. From this point of view, hybrid solutions in which different locomotion systems share common members (for example, legwheel robots based on the stepping triple-wheel concept; see Sect. 3.4.1) are better, while hybrid solutions in which two locomotion systems are never used in combination (such as the wheel-track robot in Fig. 10) are worse.

Table 2 compares the various locomotion systems with reference to the ten features discussed in Sect. 2. The first seven features describe mobility performance and are qualitatively measurable, while the last three features describe system complexity. The proposed criteria for evaluating the first seven features are shown in Table 3 with ranges corresponding to the different ranks. These criteria have been applied in Table 2.

Since mobile robots can have different sizes, some features (maximum speed, obstacle crossing capability, step/stair climbing capability, walking capability on uneven terrains) have been normalised with respect to the robot dimensions. 
Table 3. Evaluation criteria.

\begin{tabular}{|c|c|c|c|c|}
\hline \multirow[t]{2}{*}{ Feature } & \multirow[t]{2}{*}{ Evaluation criteria } & \multicolumn{3}{|c|}{ Ranges } \\
\hline & & Low & Medium & High \\
\hline maximum speed & ratio between maximum speed and overall length of robot & $<0.5 \mathrm{~s}^{-1}$ & $0.5-3 \mathrm{~s}^{-1}$ & $>3 \mathrm{~s}^{-1}$ \\
\hline $\begin{array}{l}\text { obstacle crossing } \\
\text { capability }\end{array}$ & $\begin{array}{l}\text { ratio between the maximum height of an obstacle with a } \\
\text { semi-circular lateral profile which can be crossed and the } \\
\text { height of the robot }\end{array}$ & $<0.25$ & $0.25-0.5$ & $>0.5$ \\
\hline $\begin{array}{l}\text { step/stair climbing } \\
\text { capability }\end{array}$ & $\begin{array}{l}\text { ratio between the maximum height of the square step } \\
\text { which can be crossed and the height of the robot }\end{array}$ & $<0.15$ & $0.15-0.35$ & $>0.35$ \\
\hline $\begin{array}{l}\text { slope climbing } \\
\text { capability }\end{array}$ & $\begin{array}{l}\text { maximum slope that can be climbed (compact surface, } \\
\text { friction coefficient }>0.5 \text { ) }\end{array}$ & $<15^{\circ}$ & $15-30^{\circ}$ & $>30^{\circ}$ \\
\hline $\begin{array}{l}\text { walking capability on } \\
\text { soft terrains }\end{array}$ & capability of walking on: & $\begin{array}{l}\text { compact } \\
\text { soil }\end{array}$ & $\begin{array}{l}\text { medium to } \\
\text { soft soil }\end{array}$ & soft sand \\
\hline $\begin{array}{l}\text { walking capability on } \\
\text { uneven terrains }\end{array}$ & $\begin{array}{l}\text { ratio between the maximum ground roughness that can be } \\
\text { crossed (rocky ground with random profile) and the height } \\
\text { of the robot }\end{array}$ & $<0.1$ & $0.1-0.2$ & $>0.2$ \\
\hline energy efficiency & $\begin{array}{l}\text { ratio between the final gravitational potential energy ac- } \\
\text { quired while climbing a slope at low speed and the energy } \\
\text { supplied to the actuators }\end{array}$ & $<0.1$ & $0.1-0.35$ & $>0.35$ \\
\hline
\end{tabular}

The obstacle crossing capability is defined as the capability of crossing obstacles with random shapes in unstructured environments (Table 1). Nevertheless, a semi-circular shape was selected for quantitative comparison.

As previously mentioned, locomotion systems including wheels enjoy high speed and energy efficiency. In particular, wheeled robots derived from automotive technology, featuring car-like suspensions and Ackermann steering, maximise these parameters. If not only these features were of primary importance, but obstacle crossing and climbing capabilities would also be required, wheels could be properly combined with legs in hybrid solutions. On the other hand, if task requirements are focussed on soft and yielding terrains, it is preferable to enhance robot mobility by adding tracks rather than legs, because tracks have a larger contact surface, while legs are more efficient for rigid obstacles with complex shapes.

It is important to note that slippage in the presence of yielding terrains, slopes or obstacles, which is one of the main hindrances to robot mobility, is a complex phenomenon that is remarkably influenced by the control approach (Wong, 2001). Suitable visual-based and/or adaptive control strategies can enhance the mobility performance of wheeled and tracked systems (Angelova et al., 2007; González et al., 2010). As regards legged locomotion, balance control in the presence of slippage is achieved by state-of-the-art robots with dynamic gait, such as Bigdog.

Fundamental design choice criteria are mechanical complexity and control complexity (Dudek and Jenkin, 2010).
In particular, mechanical complexity has a significant influence on robot operating dependability, in terms of both mean time between failure and mean time to repair. In this sense, pure tracked and wheeled robots are evidently simple and robust, while robots with complex mechanical designs, especially as regards the linkages in contact with the terrain (such as the leg-wheel robot in Fig. 6) should be avoided in heavy-duty applications. As regards control complexity, this is evidently higher for solutions involving legs due to gait planning requirements. Nevertheless, only these architectures, especially if controlled by model-based dynamic algorithms, can provide top performance in obstacle crossing and climbing in unstructured environments.

Starting from these general considerations, the proper mix of locomotion systems for hybrid solutions can be selected on the basis of specific task requirements.

\section{Discussion about future trends in mobile robotics}

The global market of service robotics is expected to increase substantially during the next $20 \mathrm{yr}$, surpassing the market of industrial robotics in terms of both units and sales. In this scenario, the development of efficient and flexible locomotion systems for ground mobile robots is a fundamental research issue.

As regards the future trends of mobile robotics, the most significant enabling conditions that can lead to the spread of legged mobile robots, with a level of operating flexibility that is comparable to humans and quadrupeds, concern 
the development of model-based and adaptive dynamic gait control methodologies, capable of faithfully reproducing animal behaviour. Moreover, from the hardware point of view, other important enabling conditions are the ongoing increase in the computational power of microprocessors and in the energy density of batteries, and the availability of actuators with highly specific power ratings.

Probably one of the most important technological challenges for robotics during next $10-15 \mathrm{yr}$ will be to transfer high-performance legged robots from the world of research to the world of industry and commerce by means of a difficult but necessary cost reduction process.

On the other hand, when some specific sets of features are of primary importance in the mix of operating requirements (e.g. speed, energy efficiency or mobility on yielding terrains) or when robot cost and complexity must be limited due to the economic feasibility of the application, hybrid locomotion systems are often the most suitable solutions.

For all of these reasons, the strong diversification in the current mobile robotics scenario will probably persist in the future, with a wide range of technological approaches and different levels of mechanical and control complexity.

\section{Conclusions}

The state-of-the-art of locomotion systems for mobile robots has been analysed in this paper, considering both research prototypes and industrial products, in order to provide useful indications for the early design stages, when the type of locomotion mechanism has to be selected on the basis of operating requirements.

To this end, the three main categories (wheeled, tracked and legged) and the four hybrid categories (leggedwheeled, legged-tracked, wheeled-tracked, and leggedwheeled-tracked) of locomotion systems have been compared with reference to a selection of ten features, in order to assess not only mobility performance in the most significant conditions, but also overall system complexity and reliability.

Future trends in mobile robotics are also briefly discussed. It can be said that two of the most promising lines of research are quadruped legged locomotion with dynamic gait for high-end applications in completely unstructured outdoor environments (such as military and homeland security tasks) and hybrid locomotion, especially leg-wheel and leg-track, for compact and low-cost mobile robotic platforms.

Edited by: J. Schmiedele

Reviewed by: T. Brown and another anonymous referee

\section{References}

Altendorfer, R., Moore, N., Komsuoglu, H., Buehler, M., Brown Jr., H. B., McMordie, D., Saranli, U., Full, R., and Koditschek, D. E.: RHex: a biologically inspired hexapod runner, Auton. Robot., 11, 207-213, 2001.

Angelova, A., Matthies, L., Helmick, D., and Perona, P.: Learning and prediction of slip from visual information, J. Field Robot., 24, 205-231, 2007.

Aucouturier, J. J., Ikeuchi, K., Hirukawa, H., Nakaoka, S., Shiratori, T., Kudoh, S., Kanehiro, F., Ogata, T., Kozima, H., Okuno, H. G., Michalowski, M. P., Ogai, Y., Ikegami, T., Kosuge, K., Takeda, T., and Hirata, Y.: Cheek to Chip: Dancing Robots and AI's Future, IEEE Intell. Syst., 23, 74-84, 2008.

Birk, A. and Carpin, S.: Rescue robotics: a crucial milestone on the road to autonomous systems, Adv. Robotics, 20, 596-605, 2006.

Campion, G. and Chung, W.: Wheeled robots, in: Springer Handbook of Robotics, edited by: Siciliano, B. and Khatib, O., Springer, Heidelberg, 391-410, 2008.

CCC (Computing Community Consortium) and CRA (Computing Research Association), A Roadmap for US Robotics. From Internet to Robotics, Technical report, http://www.us-robotics.us/, 2009.

Cepolina, E. E. and Hemapala, M. U.: Power tillers for demining: blast test, International Journal of Advanced Robotic Systems, 4, 253-257, 2007.

Diegel, O., Badve, A., Bright, G., Potgieter, J., and Tlale, S.: Improved Mecanum Wheel Design for Omni-directional Robots, Engineering, Proceedings of the Australasian Conference on Robotics and Automation, 117-121, 2002.

Dudek, G. and Jenkin, M.: Computational Principles of Mobile Robotics, Cambridge University Press, 2010.

EURON (EUropean RObotics research Network) and EUROP (EUropean RObotics technology Platform), The strategic research agenda for robotics in Europe, Technical report, http://www. robotics-platform.eu/sra, 2009.

Fiorini, P.: Ground Mobility Systems for Planetary Exploration, Proceedings of the IEEE International Conference on Robotics \& Automation, 908-913, 2000.

González, R., Rodríguez, F., Sánchez-Hermosilla, J., and Donaire, J. G.: Navigation Techniques for Mobile Robots in Greenhouses, Appl. Eng. Agric., 25, 153-165, 2009.

González, R., Fiacchini, M., Alamo, T., Guzman, J. L., and Rodríguez, F.: Adaptive Control for a Mobile Robot under Slip Conditions using an LMI-based Approach, Eur. J. Control, 16, 144-155, 2010.

Hamel, W. R. and Cress, R. C.: Elements of telerobotics necessary for waste clean up automation, Proc. IEEE International Conference on Robotics and Automation, 393-400, 2001.

Havlik, S.: A modular concept of the robotic vehicle for demining operations, Auton. Robot., 18, 253-262, 2005.

Hirose, S., Shirasu, T., and Fukushima, E. F.: Proposal For Cooperative Robot Gunryu Composed of Autonomous Segments, Robot. Auton. Syst., 17, 107-118, 1996.

Hirose, S., Fukushima, E., Damoto, R., and Nakamoto, H.: Design of terrain adaptive versatile crawler vehicle HELIOS-VI, Proceedings of the IEEE International Conference on Intelligent Robots and Systems, 1540-1545, 2001. 
Hirose, S., Fukuda, Y., Yoneda, K., Nagakubo, A., Tsukagoshi, H., Arikawa, K., Endo, G., Doi, T., and Hodoshima, R.: Quadruped Walking Robots at Tokyo Institute of Technology, IEEE Robotics and Automation Magazine, 16, 104-114, 2009.

Hirukawa, H., Kajita, S., Kanehiro, F., Kaneko, K., and Isozumi, T.: The Human-size Humanoid Robot That Can Walk, Lie Down and Get Up, International Journal of Robotic Research, 24, 755-769, 2005.

Hodoshima, R., Doi, T., Fukuda, Y., Hirose, S., Okamoto, T., and Mori, J.: Development of a Quadruped Walking Robot TITAN XI for Steep Slope Operation - Step Over Gait to Concrete Frames on Steep Slopes, Journal of Robotics and Mechatronics, 19, 1326, 2007.

Iagnemma, K. and Dubowsky, S.: Mobile Robots in Rough Terrain. Estimation, Motion Planning, and Control with Application to Planetary Rovers, Springer, 2004.

International Federation of Robotics, http://www.ifr.org, last access: 11 July 2012.

Kajita, S. and Espiau, B.: Legged robots, in: Springer Handbook of Robotics, edited by: Siciliano B. and Khatib, O., Springer, Heidelberg, 361-389, 2008.

Kemp, C. C., Fitzpatrick, P., Hirukawa, H., Yokoi, K., Harada, K., and Matsumoto, Y.: Humanoids, in: Springer Handbook of Robotics, edited by: Siciliano, B. and Khatib, O., Springer, Heidelberg, 1307-1333, 2008.

Kim, J., Kim, Y.-G., Kwak, J.-H., Hong, D.-H., and An, J.: Wheel \& Track Hybrid Robot Platform for Optimal Navigation in an Urban Environment, Proceedings of the SICE Annual Conference, 881-884, 2010.

Klinker, S., Lee, C. G. Y., Wagner, C., Hlawatsch, W., Schreyer, A. M., and Roser, H. P.: Destination Moon and beyond for the Micro rover Nanokhod, Proceedings of the DGLR International Symposium To Moon and beyond, 2007.

Kubotam, T., Kunii, Y., Kuroda, Y., and Working Group: Japanese lunar robotics exploration by co-operation with lander and rover, J. Earth Syst. Sci., 114, 777-785, 2005.

Lauria, M., Piquet, Y., and Siegwart, R.: OCTOPUS: an autonomous wheeled climbing robot, Proceedings of the 5th International Conference on Climbing and Walking Robots (CLAWAR), 315-322, 2002.

Lindemann, R. and Voorhees, C.: Mars exploration rover mobility assembly design, test, and performance, Proceedings of the 2005 IEEE Conference on Systems, Man and Cybernetics, 450-455, 2005.

Liu, R. and Parthasarathy, R.: Segway Human Transporter (HT): potential opportunities and challenges for transportation systems, presented at the 82nd Annual Meeting of the Transportation Research Board, Washington, DC, 2003.

Machado, J. A. T. and Silva, M. F.: An Overview of Legged Robots, Proceedings of the MME International Symposium on Mathematical Methods in Engineering, 2006.

Manchester, I. R., Mettin, U., Iida, F., and Tedrake, R.: Stable dynamic walking over uneven terrain, Int. J. Robot. Res., 30, 265 279, 2011.

McGeer, T.: Passive dynamic walking, Int. J. Robot. Res., 9, 62-82, 1990

McGhee, R. B.: Vehicular legged locomotion, in: Advances in Automation and Robotics, edited by: Saridis, G. N., JAI Press, New York, 1, 259-284, 1985
Meyer, J.-A. and Guillot, A.: Biologically inspired robots, in: Springer Handbook of Robotics, edited by: Siciliano, B. and Khatib, O., Springer, Heidelberg, 1395-1422, 2008.

Michaud, F., Letourneau, D., Arsenault, M., Bergeron, Y., Cadrin, R., Gagnon, F., Legault, M. A., Millette, M., Pare, J. F., Remblay, M. C., Lepage, P., Morin, Y., Bisson, J., and Caron, S.: MultiModal Locomotion Robotic Platform Using Leg-Track-Wheel Articulations, Auton. Robot., 18, 137-156, 2005.

Michaud, S., Schneider, A., Bertrand, R., Lamon, P., Siegwart, R., van Winnendael, M., and Schiele, A.: SOLERO: Solar Powered Exploration Rover, Proceedings of the 7th ESA Workshop on Advanced Space Technologies for Robotics and Automation, The Netherlands, 2002.

Miller, D. P., Hunt, T., Roman, M., Swindell, S., Tan, L., and Wainterholler, A.: Experiments with a Long-Range Planetary Rover, Proceedings of the International Symposium on Artificial Intelligence, Robotics and Automation in Space, 2003.

Mishkin, A.: Sojourner: an insider's view of the Mars Pathfinder mission, Berkley Books, 2004.

Morin, P. and Samson, C.: Motion control of wheeled mobile robots, in: Springer Handbook of Robotics, edited by: Siciliano, B. and Khatib, O., Springer, Heidelberg, 799-826, 2008.

Murphy, R. R.: Rescue robotics for homeland security, Communications of the ACM - Homeland security, 47, 66-68, 2004

Playter, R., Buehler, M., and Raibert, M.: Bigdog, Proceedings of the SPIE Defense\&Security Symposium, Unmanned Systems Technology, 2006.

Prassler, E. and Kosuge, K.: Domestic robotics, in: Springer Handbook of Robotics, edited by: Siciliano, B. and Khatib, O., Springer, Heidelberg, 1253-1281, 2008.

Quaglia, G., Bruzzone, L., Bozzini, G., Oderio, R., and Razzoli, R.: Epi.q-TG: mobile robot for surveillance, Industrial Robot, 38 282-291, 2011.

Quinn, R. D., Nelson, G. M., Bachmann, R. J., Kingsley, D. A., Offi, J. T., Allen, T. J., and Ritzmann, R. E.: Parallel complementary strategies for implementing biological principles into mobile robots, International Journal of Robotics Research, 22, 169-186, 2003.

Raibert, M. H.: Legged Robots That Balance, MIT Press, Cambridge, Mass, 1986.

Raibert, M. H., Brown, H. B., and Chepponis, M.: Experiments in balance with a 3D one-legged hopping machine, Int. J. Robot. Res., 3, 75-92, 1984.

Seeni, A., Schafer, B., Rebele, B., and Tolyarenko, N.: Robot mobility concepts for extraterrestrial surface exploration, Proceedings of the IEEE Aerospace Conference, 1-14, 2008.

Siegwart, R. and Nourbakhsh, I. R.: Introduction to autonomous mobile robots, MIT Press, Cambridge, 2004.

Siegwart, R., Lauria, M., Maeusli, P. A., and Van Winnendael, M.: Design and implementation of an innovative micro rover, Proceedings of Robotics 98, the 3rd Conference and Exposition on Robotics in Challenging Environments, 1998.

Siegwart, R., Lamon, P., Estier, T., Lauria, M., and Piguet, R.: Innovative Design for Wheeled Locomotion in Rough Terrain, Robot. Auton. Syst., 40, 151-162, 2002.

Snyder, R. G.: Robots assist in search and rescue efforts at WTC, IEEE Robotics and Automation Magazine, 8, 26-28, 2001.

Tadakuma, K., Tadakuma, R., Maruyama, A., Rohmer, E., Nagatani, K., Yoshida, K., Ming, A., Shimojo, M., Higashimori, M., 
and Kaneko, M.: Mechanical Design of the Wheel-Leg Hybrid Mobile Robot to Realize a Large Wheel Diameter, Proceedings of the IEEE/RSJ International Conference on Intelligent Robots and Systems, 3358-3365, 2010.

Thueer, T., Lamon P., Krebs, A., and Siegwart, R.: CRABExploration rover with advanced obstacle negotiation capabilities, Proceedings of the 9th ESA Workshop on Advanced Space Technologies for Robotics and Automation, Noordwijk, The Netherlands, 2006a.

Thueer, T., Krebs, A., and Siegwart, R.: Comprehensive Locomotion Performance Evaluation of All-Terrain Robots, Proceedings of the IEEE/RSJ International Conference on Intelligent Robots and Systems, 2006b.

Thrun, S., Montemerlo M., Dahlkamp, H., Stavens, D., Aron, A., Diebel, J., Fong, P., Gale, J., Halpenny, M., Hoffmann, G., Lau, K., Oakley, C., Palatucci, M., Pratt, V., Stang, P., Strohband, S., Dupont, C., Jendrossek, L.-E., Koelen, C., Markey, C., Rummel, C., van Niekerk, J., Jensen, E., Alessandrini, P., Bradski, G., Davies, B., Ettinger, S., Kaehler, A., Nefian, A., and Mahoney, P.: STANLEY, the robot that won the DARPA Gran Challenge, J. Field Robot., 23, 661-692, 2006.
Van Straten, G., van Willigenburg, L. G., and Tap, R. F.: The Significance of Crop Co-States for Receding Horizon Optimal Control of Greenhouse Climate, Control Eng. Pract., 10, 625-632, 2002.

Vukobratovic, M. and Borovac, B.: Zero-moment point- thirty five years of its life, Int. J. Hum. Robot., 1, 157-173, 2004.

Wong, J. Y.: Theory of Ground Vehicles, John Wiley \& Sons, 2001.

Woosub, L., Sungchul, K., Munsang, K., and Mignon, P.: ROBHAZ-DT3: teleoperated mobile platform with passively adaptive double-track for hazardous environment applications, Proceedings of the IEEE/RSJ International Conference on Intelligent Robots and Systems, 1, 33-38, 2004.

Yi, J., Song, D., Levandowski, A., and Jayasuriya, S.: Trajectory tracking and balance stabilization control of autonomous motorcycles, Proceedings of the IEEE International Conference on Robotics and Automation ICRA 2006, 2583-2589, 2006.

Yokota, S., Kawabata, K., Blazevic, P., and Kobayashi, H.: Control Law for Rough Terrain Robot with Leg-type Crawler, Proceedings of the IEEE International Conference on Mechatronics and Automation, 2006. 